\title{
Effect of Fish Oil on Heart Health: A Meta-Analysis
}

\author{
Hannah Guan* \\ Division of Biostatistics, University of Minnesota, USA \\ *Corresponding author: Hannah Guan, Division of Biostatistics, University of Minnesota, 420 Delaware St SE, Minneapolis, \\ MN 55455, USA
}

\section{ARTICLE INFO}

Received: 幽 February 17, 2021

Published: March 05, 2021

Citation: Hannah Guan. Effect of Fish Oil on Heart Health: A Meta-Analysis. Biomed J Sci \& Tech Res 34(3)-2021. BJSTR. MS.ID.005543.

Keywords: Meta-Analysis; Fatty Acid; Cardiac Death

Abbreviations: EPA: Eicosapentaenoic Acid; DHA: Docosahexaenoic Acid; AHA: American Heart Association; CVDs: Cardiovascular Diseases; RRs: Relative Risks; CI: Confidence Interval

\begin{abstract}
A fatty acid is a $\mathrm{CO} 2 \mathrm{H}$ compound with a long aliphatic chain, which yields large quantities of ATP (Adenosine Triphsophate) when metabolized, making them an important dietary source of fuel for humans. Fatty acids cannot be made by human body and have to be obtained from dietary fats. Consuming a diet low in saturated fatty acids and high in unsaturated fatty acids may potentially lower the risk of cardiovascular diseases, diabetes, and death. Fish oil is an important source of unsaturated fatty acids, primarily containing the omega-3 fatty acids eicosapentaenoic acid (EPA) and docosahexaenoic acid (DHA). The American Heart Association (AHA) recommends fish and fish oil supplements as part of a healthy diet. However, there have been ongoing debates about whether fish oil is actually beneficial to the heart. In this project, a literature search was conducted on public database for existing studies on intake of fish oil or omega- 3 fatty acids and cardiovascular health, specifically, cardiac death. A total of 20 relevant studies were found until 2018. A meta-analysis was used to compare these published results and their heterogeneity. A pooled estimate of relative risk was 0.89 (95\% confidence interval: $[0.81,0.97], p$-value $=0.006)$, suggesting that intake of omega- 3 fatty acids can lower the risk of cardiac death by $11 \%$. Heterogeneity across study results were not statistically significant $(p$-value $=0.11)$. These results support the AHA recommendation on fish oil supplement in diet.
\end{abstract}

\section{Introduction}

A fatty acid is a $\mathrm{CO} 2 \mathrm{H}$ compound, called carboxylic acid, with a long aliphatic chain, a compound that doesn't have any rings and can either be branched or straight. Fatty acids yield large quantities of ATP when metabolized, making them an important dietary source of fuel for humans. One can get fatty acids from dietary fats, such as butter, duck fat, and coconut oil. Fatty acids are split into two groups, saturated and unsaturated. Saturated fatty acids are fatty acids that only have single bonds. Consuming a diet low in saturated fatty acids may potentially lower the risk of cardiovascular diseases, diabetes, and death [1-5]. Unsaturated fatty acids have more than one double bond. The two types of un-saturated fatty acids are monounsaturated fatty acids, MUFAs (Monounsaturated Fat Acid), and polyunsaturated fatty acids, PUFAs (Polyunsaturated Fatty Acid). MUFAs only have one double bond and the rest are single bonds, while PUFAs contain more than one double bond. MUFA is known to protect against cardiovascular disease by providing more membrane fluidity than saturated fats [6]. A few sources of MUFA are olive oil, dry roasted pecans, and beef. The effect of
MUFA and PUFA are very similar, but PUFAs decrease the number of triglycerides a little more. Fish oil is an important source of PUFA, primarily containing the omega- 3 fatty acids eicosapentaenoic acid (EPA) and docosahexaenoic acid (DHA) [3,7].

The American Heart Association (AHA) recommends fish and fish oil supplements as part of a healthy diet because the human body is not able to make these fatty acids by itself [8]. However, there have been ongoing debates about whether fish oil is actually beneficial to the heart [9]. Many studies have been conducted, including observational studies or randomized clinical trials, to test for association between intake of fish oil or other forms of omega-3 fatty acids supplements and cardiovascular outcomes, and the results are mixed [10-15]. The inconsistency of results may be due to difference in target populations, such as healthy vs high-risk population, limitation on sample size, amount of intake, and choice of outcomes. Meta-analysis is a statistical technique that combines results from multiple studies. Compared to a single study, me-ta-analysis not only improves statistical power by 
aggregating information from many studies, but also leads to more robust estimate, quantifies heterogeneity of studies, and evaluates potential publication bias. In this project, a literature search will be conducted for existing studies to answer the question whether fish oil (or omega-3 fatty acids) is beneficial to cardiovascular health. A meta-analysis will be performed on selected studies to compare the results and identify pattern across studies. Among different types of cardiovascular outcomes, cardiac death is used as the primary outcome. The null hypothesis of this study is that the intake of fish oil or omega-3 fatty acid is not associated with cardiac death.

\section{Materials and Methods}

A comprehensive literature search was conducted on the PubMed database (https://www.ncbi.nlm.nih.gov/pubmed) to search for publications or studies about the effect of omega 3 fatty acids on cardiovascular health. Since cardiovascular health is a very broad topic, including many types of cardiovascular diseases (CVDs), such as heart attacks, stroke, and others, the studies were narrowed down to ones with cardiac deaths to make the results more comparable. The data for this project were primarily from two major reviews published recently between 2016 and 2018 . The first is a literature review by Rizos, et al. [16], summarizing 21 studies from PubMed, all of which were published by 2018. Most of the studies in this review concluded that omega-3 has no significant benefit on cardiovascular health, except 2 studies reported significant association with cardiac death, and 2 studies reported association with other cardiovascular events. Overall, this paper summarizes that a regular consumption of omega 3 PUFAS can be healthy, but it has no significant effect that benefits CVDs. The second is a meta-analysis performed by Maki, et al. [17], using 14 studies published from January 1947 to December 2016 found on the PubMed and Ovid/MEDLINE databases. The conclusion of the me-ta-analysis was that omega-3 supplementation is responsible for some benefits in cardiac health.

For cardiac death, a pooled relative risk of 0.92 was estimated from these studies, i.e., risk of cardiac death in omega- 3 group is $8 \%$ lower than that in non-omega-3 group. Studies from these two reviews were carefully evaluated to establish a comprehensive collection of studies between omega-3 fatty and cardiac death.
Further search was conducted on PubMed, using keywords "cardiac death" and ("omega-3 fatty acid" or "fish oil"), and one large-scale cohort study was published in July of 2017 [18]. At first, a fixedeffects meta-analysis was conducted to compare risk of cardiac death between the omega-3 (or fish oil) group and the control group. Relative risks (RRs) were reported from individual studies or calculated manually based on number of events in the two groups. Studies were inversely weighted by estimated variances of relative risks. A pooled estimate and its $95 \%$ confidence interval (CI) were calculated. Statistical significance was declared for $\mathrm{p}$-value $<0.05$, or the null risk value 1.0 was not included in the $95 \%$ CI. Between study variation among individual results was assessed using Cochran's Q test, and a p-value of 0.05 or less would indicate significant heterogeneity. In addition, the I2 (I-square) statistic was calculated, which represents the percentage of variation attributable to between-study hetero-geneity.

Later, a random-effects meta-analysis was also conducted because it is more robust to study heterogeneity, while fixed-effects model assumes all studies from the same population and having exactly the same effect size. Forest plot was generated as a visual summary of individual and pooled results. Funnel plot was used to examine potential public-cation bias, which is a common problem in meta-analysis where insignificant findings might be less likely reported in scientific journals.

\section{Data}

A total of 20 studies were included in the meta-analysis that reported association results between fish oil or omega-3 intakes and cardiac death. Summary characteristics of individual studies were shown in (Table 1). There were 102,426 participants combined from these studies, including 5,277 cardiac deaths. Most of the participants were recruited from United States or European countries, except one study from Japan and one from India. The follow-up time of each study varied from 0.5 to 19 years. Most of studies used either fish oil or Ethyl esters as form of omega-3 fatty acids, with dose varying between 0.2 and 6 gram/day. Only three of the 20 studies showed significant association between omega- 3 fatty acid or fish oil intake and cardiac death ( $\mathrm{p}<0.05)$, but 13 studies showed positive effect for omega-3 fatty acid (RR $<1)$.

Table 1: Characteristics of selected studies.

\begin{tabular}{|c|c|c|c|c|c|c|c|c|c|}
\hline Study & Author & Year & Country & $\begin{array}{c}\text { Total } \\
\text { Subjects }\end{array}$ & $\begin{array}{c}\text { Relative } \\
\text { Risk }\end{array}$ & P Value & $\begin{array}{c}\text { Duration } \\
\text { (Years) }\end{array}$ & $\begin{array}{c}\text { Dosage } \\
\text { Agent }\end{array}$ \\
\hline HARP & Sacks & 1995 & USA & 59 & 0.30 & 0.46 & 2.3 & Fish oil & 6 \\
\hline IEIS-4 & Singh & 1997 & India & 240 & 0.52 & 0.033 & 1 & Fish oil & 6 \\
\hline Leng & Leng & 1998 & UK & 120 & 1 & 1.00 & 2 & Ethyl esters & 6 \\
\hline GISSI & Marchioli & 1999 & Italy & 11,324 & 0.78 & 0.004 & 3.5 & Ethyl esters & 1 \\
\hline CART & Johansen & 1999 & Norway & 388 & 0.33 & 0.33 & 0.5 & Ethyl esters \\
\hline SICMO & Von Schacky & 1999 & Germany & 223 & 0.33 & 0.50 & 2 & Fish oil & 6 \\
\hline Nilsen & Nilsen & 2001 & Norway & 300 & 1 & 1.00 & 1.5 & Ethyl ester & 4 \\
\hline Leaf & Leaf & 2005 & USA & 402 & 1 & 1.00 & 1 & Fish oil & 2.6 \\
\hline
\end{tabular}




\begin{tabular}{|c|c|c|c|c|c|c|c|c|c|}
\hline OPACH & Svensson & 2006 & Denmark & 206 & 1.12 & 0.66 & 2 & Ethyl esters & 1.7 \\
\hline SOFA & Brouwer & 2006 & Multiple ${ }^{1}$ & 546 & 0.46 & 0.11 & 1 & Fish Oil & 0.96 \\
\hline JELIS & Yokoyama & 2007 & Japan & 18,645 & 0.94 & 0.79 & 4.6 & Ethyl esters & 1.84 \\
\hline GISSI-HF & Tavazzi & 2008 & Italy & 6,975 & 0.92 & 0.12 & 3.9 & Ethyl esters & 1 \\
\hline OMEGA & Rauch & 2010 & Germany & 3,818 & 0.95 & 0.84 & 1 & Ethyl esters & 1 \\
\hline DOIT & Einvik & 2010 & Norway & 563 & 0.43 & 0.21 & 3 & Fish oil & 4 \\
\hline SU.FOL.OM3 & Galan & 2010 & France & 2,501 & 1.03 & 0.88 & 4.7 & Fatty acid & capsule \\
\hline Alpha Omega & Kromhout & 2010 & Netherlands & 4,837 & 0.95 & 0.75 & 3.4 & Margarines & 0.2 \\
\hline ORIGIN & Bosch & 2012 & Multiple & 12,536 & 0.98 & 0.77 & 6.2 & Ethyl esters & 1 \\
\hline Risk \& Prev & Roncaglioni & 2013 & Italy & 12,505 & 1.07 & 0.64 & 5 & Ethyl esters & 1 \\
\hline AREDS2 & Bonds & 2014 & USA & 4,203 & 1.39 & 0.58 & 4.8 & Ethyl esters & 1 \\
\hline EPIC-Norfolk & Lentjes & 2017 & Uk & 22,035 & 0.74 & $<0.001$ & 19 & Any supplement & 3 \\
\hline
\end{tabular}

\section{Note:}

1. Poland, Germany, Switzerland, UK, Czech Republic, Austria, Belgium, Netherlands.

2. An international study with participants from North and South America, Europe, China, India, and Australia.

\section{Results}

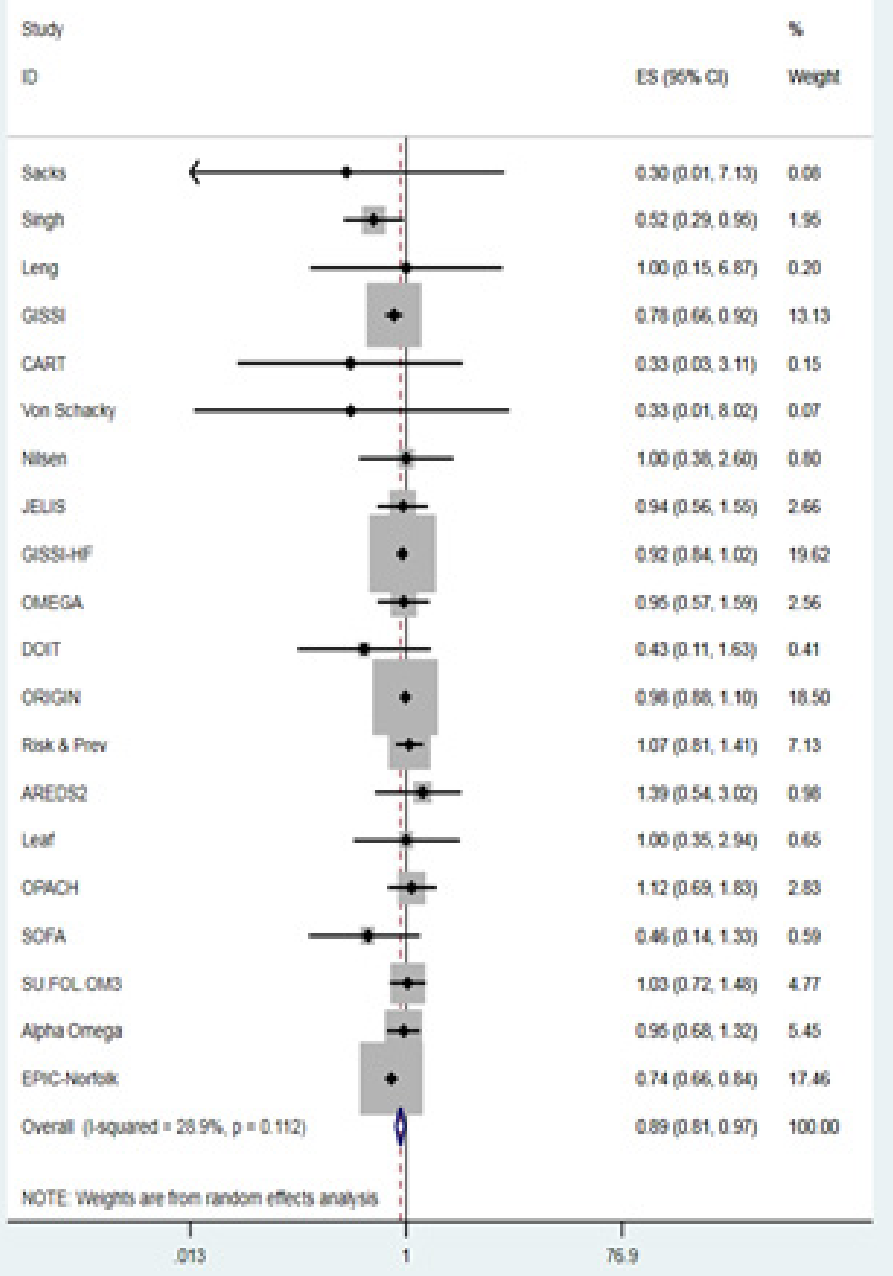

Figure 1: Forest plots derived from random-effects meta-analysis model. 
A fixed-effect meta-analysis estimated a pooled relative risk of 0.86 (95\% CI: 0.81-0.91, p-value<0.001), suggesting that intake of omega-3 fatty acids can significantly lower the risk of cardiac death by $14 \%$. A forest plot is presented in (Figure 1). Although the studies differed in many aspects, for example, population, followup duration, and dose of omega-3 fat-ty acids, no significant study heterogeneity was observed (Cochran's $Q$ test $p$-value $=0.11$ ). The I2 (I-square) value of $28.9 \%$ indicated that only a small amount of variability between studies is due to heterogeneity. To obtain robust association, a random-effect meta-analysis was performed, leading to a pooled estimate of RR (Relative Risk) of 0.89 (95\% CI: $0.81-0.97, \mathrm{p}$-value $=0.006$ ). As most of the studies recruited participants from US or European countries, a subgroup analysis was run, which ex-cluded IEIS-4, JELIS, and ORIGIN study. The pooled estimate from fixed-effect model remained almost the same $(\mathrm{RR}=0.86,95 \% \mathrm{CI}: 0.81-0.92)$, but the $\mathrm{I} 2$ index decreased from $28.9 \%$ to $12.7 \%$ (Figure 2). It suggests that part of the study het erogeneity is likely from difference in study populations. To assess potential publication bias, a funnel plot (Figure 3) was shown for the relationship between estimated effect sizes (loge(RR)) and their precision (standard errors of loge(RR)). No obvious asymmetricity was observed, suggesting no apparent publication bias.

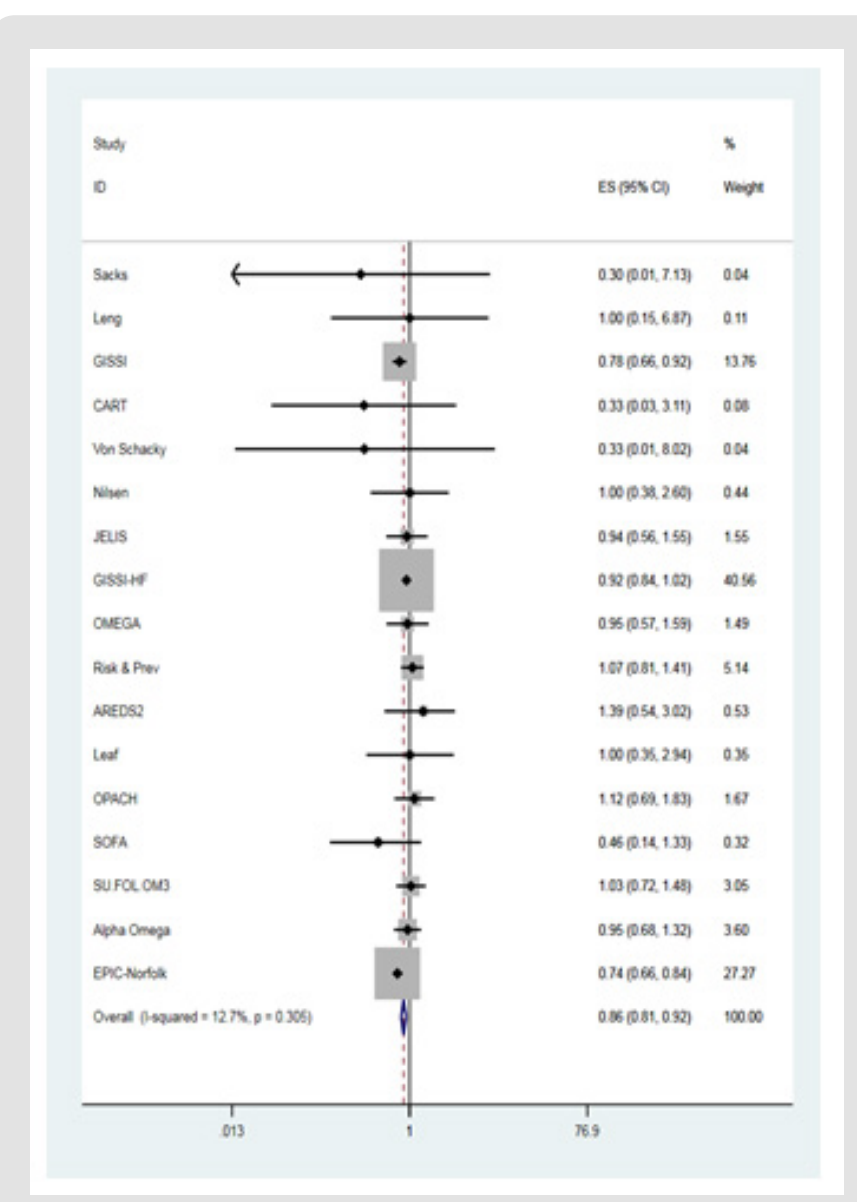

Figure 2: Forest plot for US- or European-based studies.

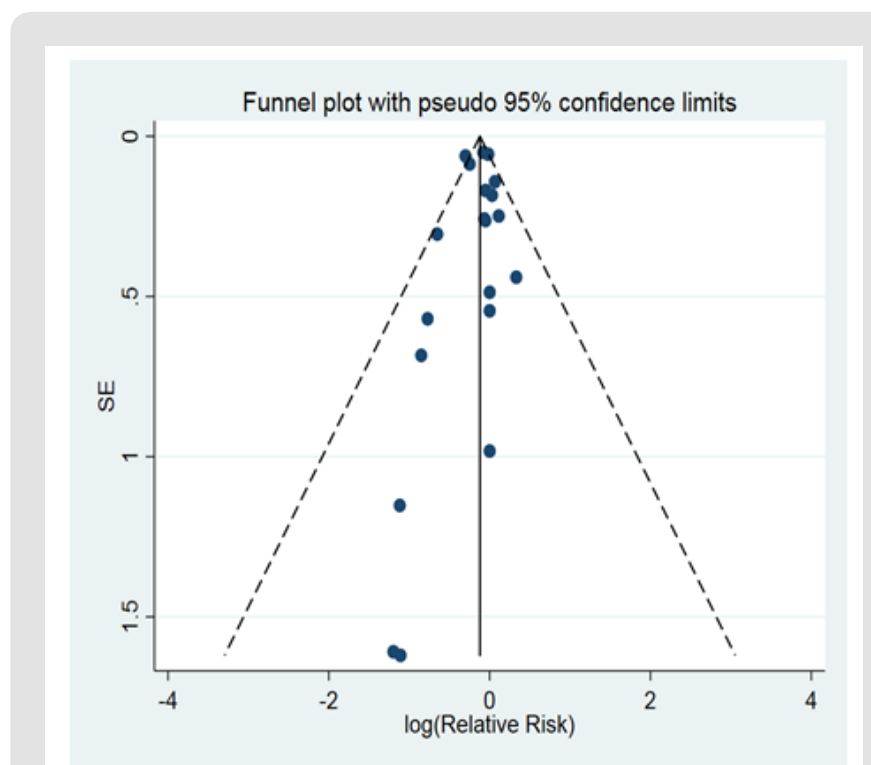

Figure 3: Funnel plot of standard error (SE) by $\log$ (relative risk) for publication bias.

\section{Conclusion and Future Work}

In this project, a meta-analysis was conducted to evaluate relationship between intake of fish oil or omega-3 fatty acids and cardiac death, based on twenty published studies until December of 2017. Significant positive effect of omega-3 fatty acids was estimated when pooling these individual study results. This finding is consistent with recent AHA recommendation that dietary fish oil intake can be beneficial to cardiovascular health, although the effect may be small. Results from this project suggests the beneficial role of omega-3 fatty acid or fish oil on cardiac death. Although the effect size is relatively small ( $\sim 14 \%$ reduction in risk), from a view of public health, the benefit can still be meaningful when applied to large population. Also, given the low risk for adverse effects, dietary intake of omega-3 fatty acid should be encouraged. In future studies, other cardiovascular events, such as MI (Myocardial Infarction, commonly known as a heart attack) and stroke, can be considered in addition to cardiac death.

\section{Funding}

This research received no external funding.

\section{Institutional Review Board Statement}

Not applicable.

\section{Informed Consent Statement}

Not applicable.

\section{Data Availability Statement}

The data presented in this study are available upon request to the author. 


\section{Conflicts of Interest}

The authors declare no conflict of interest.

\section{References}

1. Sacks FM, Lichtenstein AH, Wu HY, Appel LJ, Creager MA, et al. (2017) Dietary fats and cardiovascular disease: A presidential advisory from the american heart association. Circulation 136(3): 1-12.

2. Hooper L, Martin N, Abdelhamid A, Smith GD (2015) Reduction in saturated fat intake for cardiovascular disease. Cochrane Database Syst Rev 6: CD011737.

3. Siscovick DS, Barringer TA, Fretts AM, Wu JH, Lichtenstein AH, et al. (2017) Omega-3 polyunsaturated fatty acid (fish oil) supplementation and the prevention of clinical cardiovascular disease. Circulation 135: e867-e884.

4. Fard SG, Wang F, Sinclair AJ, Elliott G, Turchini GM (2019) How does high DHA fish oil affect health? A systematic review of evidence. Critical Reviews in Food Science and Nutrition 59(11): 1684-1727.

5. Ajith TA, Jayakumar TG (2018) Omega-3 fatty acids in coronary heart disease: Recent updates and future perspectives. Clinical and Experimental Pharmacology and Physiology 46(1): 11-18.

6. Kris Etherton PM (1999) Monounsaturated fatty acids and risk of cardiovascular disease. Circulation 100(11): 1253-1258.

7. Durmus M (2018) Fish oil for human health: Omega-3 fatty acid profiles of marine seafood species. Food Science and Tech-nology.

8. Mengelberga A, Leathema J, Poddb J (2018) Fish oil supplement use in New Zealand: A cross-sectional survey. Complementary Therapies in Clinical Practice 33: 118-123.

9. Brinson BE, Miller S (2012) Fish oil: What is the role in cardiovascular health? Journal of Pharmacy Practice 25(1): 69-74.

\section{ISSN: 2574-1241}

DOI: 10.26717/BJSTR.2021.34.005543

Hannah Guan. Biomed J Sci \& Tech Res

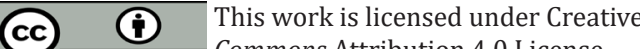

Commons Attribution 4.0 License

Submission Link: https://biomedres.us/submit-manuscript.php
10. Tummala R, Ghosh RK, Jain V, Devanabanda RA, Bandyopadhyay D, et al. (2019) Fish oil and cardiometabolic diseases: Recent updates and controversies. The American Journal of Medicine 132(10): 1153-1159.

11. Papaioannou V, Pnevmatikos First I (2019) Heart rate variability: A potential tool for monitoring immunomodulatory effects of parenteral fish oil feeding in patients with Sepsis. Nutrition and Metabolic Insights 12: 1178638819847486

12. Goel A, Pothineni NV, Singhal M, Paydak H, Saldeen T, et al. (2018) Fish, fish oils and cardio protection: Promise or fish tale? International Journal of Molecular Sciences, 19(12): 3703.

13. Nejm MB, Haidar AA, Hirata AE, Oyama LM, Guimarães de Almeida AC, et al. (2017) Fish oil supplementation reduces heart levels of interleukin-6 in rats with chronic inflammation due to epilepsy. Frontiers in Neurology 8: 263.

14. Lin Z, Chen R, Jiang Y, Xia Y, Niu Y, et al. (2019) Cardiovascular benefits of fish-oil supplementation against fine particulate air pollution in China. Journal of the American College of Cardiology 73(16): 2076-2085.

15. Chu Z, Wei Y, Hao Y, Wang J, Hang F, et al. (2020) Clinical effectiveness of fish oil on arterial stiffness: A sys-tematic review and meta-analysis of randomized controlled trials. Nutrition, Metabolism and Cardiovascular Diseases.

16. Evangelos CR, Elisaf MS (2017) Does supplementation with omega-3 PUFAs add to the prevention of cardiovascular disease? Current Cardiology Reports 19(6): 47

17. Maki KC, Palacios OM, Bell M, Toth PP (2017) Use of supplemental long-chain omega-3 fatty acids and risk for cardiac death: An updated meta-analysis and review of research gaps. Journal of Clinical Lipidology 11(5): 1152-1160.

18. Lentjes MAH, Ruth HK, Welch AA, Mulligan AA, Luben RN, et al. (2017) Longitudinal associations between marine omega-3 supplement users and coronary heart disease in a UK population-based cohort. BMJ Open 7(10): e017471.

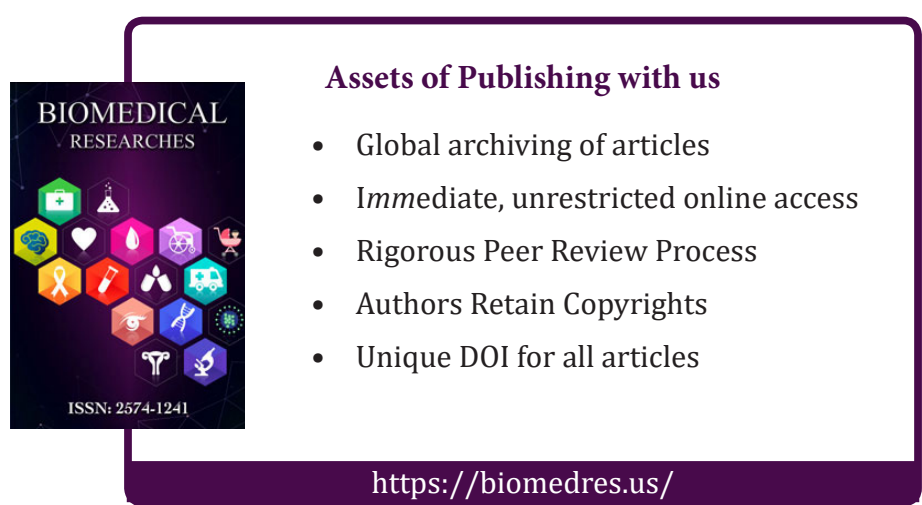

